\title{
Search for Quadrupole Strength in the Electroexcitation of the $\Delta^{+}(1232)$
}

C. Mertz, ${ }^{1,2}$ C. E. Vellidis, ${ }^{2}$ R. Alarcon, ${ }^{1}$ D. H. Barkhuff, ${ }^{3}$ A. M. Bernstein, ${ }^{4}$ W. Bertozzi, ${ }^{4}$ V. Burkert,${ }^{5}$ J. Chen, ${ }^{4}$ J. R. Comfort, ${ }^{1}$ G. Dodson, ${ }^{4}$ S. Dolfini, ${ }^{1}$ K. Dow ${ }^{4}$ M. Farkhondeh, ${ }^{4}$ J. M. Finn,${ }^{6}$ S. Gilad,${ }^{4}$ R. W. Gothe, ${ }^{7}$ X. Jiang, ${ }^{8}$ K. Joo, ${ }^{4}$ N. I. Kaloskamis, ${ }^{2,4}$ A. Karabarbounis, ${ }^{2}$ J. J. Kelly, ${ }^{9}$ S. Kowalski, ${ }^{4}$ C. Kunz, ${ }^{7}$, R. W. Lourie, ${ }^{3}$ J. I. McIntyre, ${ }^{6}$ B. D. Milbrath, ${ }^{3}$ R. Miskimen, ${ }^{8}$ J. H. Mitchell, ${ }^{5}$ C. N. Papanicolas, ${ }^{2}$ C. F. Perdrisat, ${ }^{6}$ A. J. Sarty, ${ }^{10}$ J. Shaw, ${ }^{8}$ S.-B. Soong, ${ }^{4}$ D. Tieger, ${ }^{4}$ C. Tschalær, ${ }^{4}$ W. Turchinetz, ${ }^{4}$ P. E. Ulmer, ${ }^{11}$ S. Van Verst, ${ }^{4}$ G. A. Warren, ${ }^{4}$ L. B. Weinstein, ${ }^{11}$ S. Williamson, ${ }^{12}$ R. J. Woo, ${ }^{6}$ and A. Young ${ }^{1}$

${ }^{1}$ Department of Physics and Astronomy, Arizona State University, Tempe, Arizona 85287

${ }^{2}$ Institute of Accelerating Systems and Applications and Department of Physics, University of Athens, Athens, Greece ${ }^{3}$ Institute for Nuclear and Particle Physics and Department of Physics, University of Virginia, Charlottesville, Virginia 22901

${ }^{4}$ Department of Physics, Laboratory for Nuclear Science and Bates Accelerator Center, Massachusetts Institute of Technology, Cambridge, Massachusetts 02139

${ }^{5}$ Thomas Jefferson National Accelerator Facility, Newport News, Virginia 23606

${ }^{6}$ Physics Department, College of William and Mary, Williamsburg, Virginia 23187

${ }^{7}$ Department of Physics, Bonn University, Bonn, Germany

${ }^{8}$ Department of Physics, University of Massachusetts, Amherst, Massachusetts 01003

${ }^{9}$ Department of Physics, University of Maryland, College Park, Maryland 20742

${ }^{10}$ Department of Physics, Florida State University, Tallahasse, Florida 32306

${ }^{11}$ Department of Physics, Old Dominion University, Norfolk, Virginia 23529

${ }^{12}$ Physics Department, University of Illinois, Urbana-Champaign, Urbana, Illinois 61801

(Received 13 November 2000)

High-precision ${ }^{1} \mathrm{H}\left(e, e^{\prime} p\right) \pi^{0}$ measurements at $Q^{2}=0.126(\mathrm{GeV} / c)^{2}$ are reported, which allow the determination of quadrupole amplitudes in the $\gamma^{*} N \rightarrow \Delta$ transition; they simultaneously test the reliability of electroproduction models. The derived quadrupole-to-dipole $(I=3 / 2)$ amplitude ratios, $R_{\mathrm{SM}}=\left(-6.5 \pm 0.2_{\text {stat }+ \text { sys }} \pm 2.5_{\text {mod }}\right) \%$ and $R_{\mathrm{EM}}=\left(-2.1 \pm 0.2_{\text {stat }+ \text { sys }} \pm 2.0_{\text {mod }}\right) \%$, are dominated by model error. Previous $R_{\mathrm{SM}}$ and $R_{\mathrm{EM}}$ results should be reconsidered after the model uncertainties associated with the method of their extraction are taken into account.

DOI: 10.1103/PhysRevLett.86.2963

PACS numbers: 14.20.Gk, 13.60.Le, 25.30.Rw

The conjecture that the nucleon is deformed, raised more than 20 years ago [1], continues to be the subject of intense theoretical [2-8] and experimental [9-15] activity. Because the quadrupole moment of the nucleon vanishes on account of its spin- $1 / 2$ nature, this investigation has naturally turned to the search for quadrupole strength in nucleon resonances, specifically, in the $\gamma^{*} N \rightarrow \Delta(1232)$ transition.

Spin-parity selection rules in the $N\left(1 / 2^{+}\right) \rightarrow \Delta\left(3 / 2^{+}\right)$ transition allow magnetic dipole $(M 1)$ and electric $(E 2)$ or Coulomb quadrupole $(C 2)$ amplitudes. In the naive (spherical) quark model of the nucleon, the $\Delta$ excitation is a pure spin-flip (M1) transition. Experimentally, $M 1$ is indeed found to dominate. In more refined models, small $E 2$ and $C 2$ amplitudes are predicted. The physical origin of these contributions is attributed to different mechanisms in the various models; they have important implications for our understanding of the structure of the nucleon and of QCD at low energies [2-5]. For instance, in "QCDinspired" constituent quark models, nonvanishing $E 2$ and $C 2$ components arise from intraquark effective colormagnetic tensor forces [3] whereas, in "cloudy" baryon models, they arise mostly from meson exchange currents [5].
In pion production, the multipoles are denoted by $M_{l \pm}^{I}$, $E_{l \pm}^{I}$, and $S_{l \pm}^{I}$, indicating their character (magnetic, electric, or scalar), their isospin (I), and their total angular momentum $(J=l \pm 1 / 2)$. Thus, the resonant photon multipoles $M 1, E 2$, and $C 2$ correspond to $M_{1+}^{3 / 2}, E_{1+}^{3 / 2}$, and $S_{1+}^{3 / 2}$, respectively. The electric- and scalar-to-magneticamplitude-ratio are defined as $R_{\mathrm{EM}}=\operatorname{Re}\left(E_{1+}^{3 / 2} / M_{1+}^{3 / 2}\right)$ and $R_{\mathrm{SM}}=\operatorname{Re}\left(S_{1+}^{3 / 2} / M_{1+}^{3 / 2}\right)$, respectively. Most models of the nucleon have definite predictions for these ratios. They are very small at low momentum transfers, the domain of the reported measurements. The predictions for $R_{\mathrm{EM}}$ at $Q^{2}=0$ range from $-0.1 \%$ up to $-5 \%$ [2-5].

While $R_{\mathrm{EM}}$ measurements at $Q^{2}=0$ are pursued with the use of real photons, its $Q^{2}$ evolution and the $R_{\mathrm{SM}}$ ratio can be investigated only through electroexcitation. A number of calculations explore the dependence of $R_{\mathrm{EM}}$ and $R_{\mathrm{SM}}$ on $Q^{2}$ [2,6-8]. The experimental determination of $R_{\mathrm{EM}}$ and $R_{\mathrm{SM}}$ is severely complicated by the presence of nonresonant processes that are coherent with the resonant excitation of the $\Delta(1232)$ [16]. These processes (such as nucleon and pion pole contributions, $u$-channel nucleon and $\Delta$ exchange, and tails of higher resonances), termed "background contributions," need to be constrained with 
model calculations and measurements tailored to this end. Also, it is imperative that electroproduction models, used in model extraction of $R_{\mathrm{EM}}$ and $R_{\mathrm{SM}}$, are adequately tested in their ability to accurately handle small amplitudes, both resonant and background.

Precision measurements with polarized tagged photons have resulted in an $R_{\mathrm{EM}}$ at a resonance of $(-3.0 \pm 0.3) \%$ [9] and $(-2.5 \pm 0.3) \%$ [10]. Model calculations are in reasonably good agreement with experiment [17-19]. The situation is quite different for electron scattering investigations. Experiments conducted in the late 1960's and early 1970's for $Q^{2}$ up to $1(\mathrm{GeV} / c)^{2}$ have yielded $R_{\mathrm{EM}}$ values consistent with zero and $R_{\mathrm{SM}}$ of around $-7 \%$ with large statistical and systematic errors [11-13]. A dispersion relation analysis [20] reported exceptionally large values of $R_{\mathrm{SM}}$ around $-13 \%$ in the range of $Q^{2}=$ $0.1-0.25(\mathrm{GeV} / c)^{2}$, suggestive of a narrow structure peaking near $Q^{2}=0.1(\mathrm{GeV} / c)^{2}$. These values are consistent with the value $\operatorname{Re}\left(S_{1+} / M_{1+}\right)=(-12.7 \pm 1.5) \%$ of the ratio of isospin-mixed multipoles reported in a recent $\mathrm{H}\left(e, e^{\prime} \pi^{0}\right) p$ experiment at $Q^{2}=0.127(\mathrm{GeV} / c)^{2}$ [14]. The measurements reported here, performed at the same $Q^{2}$, allow a direct comparison with the aforementioned data.

The coincident $\mathrm{H}\left(e, e^{\prime} p\right) \pi^{0}$ cross section in the onephoton-exchange-approximation can be written as [21]

$$
\begin{aligned}
\frac{d \sigma}{d \omega d \Omega_{e} d \Omega_{p q}^{c m}}= & \Gamma_{v} \frac{p_{c m}}{k_{c m}} \sigma, \\
\sigma= & R_{T}+\varepsilon_{L} R_{L}-\rho_{L T} R_{L T} \cos \phi \\
& +\varepsilon R_{T T} \cos 2 \phi,
\end{aligned}
$$

where $\Gamma_{v}$ is the virtual photon flux; $p_{c m}$ and $k_{c m}$ are the pion momentum and the photon equivalent energy in the hadronic CM frame, respectively; $\varepsilon, \varepsilon_{L}$, and $\rho_{L T}$ are electron kinematic factors; and $\phi$ is the nucleon azimuthal angle about the momentum transfer $\vec{q}$ measured from the nucleon direction closest to the beam exit line. $R_{L}, R_{T}$, $R_{L T}$, and $R_{T T}$ are the longitudinal, transverse, longitudinaltransverse, and transverse-transverse interference response functions, respectively [21].

To study the $\gamma^{*} N \rightarrow \Delta$ transition with high precision, an extensive program has been developed at the MIT-Bates Linear Accelerator. We report here results from the first phase of the program. We have reported the recoil proton polarization $P_{n}$ result from the same experiment [22].

The experiment $[22,23]$ was conducted at energies of 719 and $799 \mathrm{MeV}$ and a liquid $\mathrm{H}_{2}$ target was used; the scattered electrons were detected in the "MEPS" spectrometer and the coincident protons in "OHIPS". The focal plane instrumentation of each spectrometer consisted of one crossed vertical drift chamber for track reconstruction and scintillators for triggering. Detailed optics studies were done for each spectrometer, and the detection efficiencies were measured as functions of all independent reaction coordinates. The phase-space normalization of the cross section and various corrections applied to the data, including radiative corrections, were implemented with the aid of a Monte Carlo simulation model. The coincident cross section was measured at $\phi=0$ and $\pi$ for a broad range of hadronic mass $W$ around the resonance and a range of proton polar angle $\theta$ about $\vec{q}$ in the hadronic CM frame near $\theta=0$.

Figure 1 shows the coincident cross section as a function of the hadronic mass $W$ for proton detection at $\theta=0$, where $R_{L T}$ and $R_{T T}$ vanish and $R_{T}$ has the maximum sensitivity to $\operatorname{Re}\left(E_{1+}^{*} M_{1+}\right)$. The data exhibit a distinct resonant shape, arising mostly from $\left|M_{1+}\right|^{2}$. Figure 2 shows the response function $R_{L T}$ and the cross section asymmetry $A_{L T}$ which are sensitive to $\operatorname{Re}\left(S_{1+}^{*} M_{1+}\right)$,

$$
A_{L T}=\frac{\sigma_{\phi=0}-\sigma_{\phi=\pi}}{\sigma_{\phi=0}+\sigma_{\phi=\pi}}=\frac{-\rho_{L T} R_{L T}}{R_{T}+\varepsilon_{L} R_{L}+\varepsilon R_{T T}} .
$$

The measured cross section (Fig. 1), asymmetry, and $R_{L T}$ response function (Fig. 2), are compared with the curves that result by adjusting the relevant parameters in the models of Drechsel et al. [17,24] (MAID), of Sato and Lee (SL) [18,25], and of Davidson and Mukhopadhyay (RPI [Rensselaer Polytechnic Institute]) [19,26]. All three models start from the same Lagrangian for the nonresonant terms, including explicit nucleon and light meson $(\pi$, $\rho, \omega)$ degrees of freedom coupled to the electromagnetic field. Their principal differences lie in the definition of the

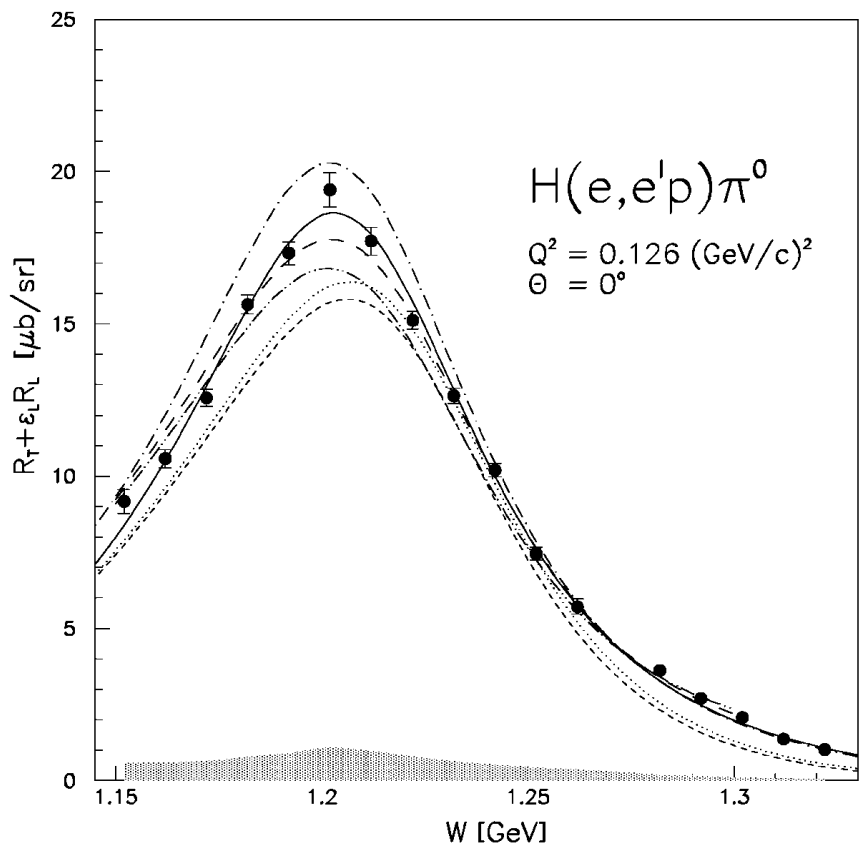

FIG. 1. The CM cross section in parallel kinematics. The curves are as follows: solid: fit of MAID [17,24]; dot-dashed: corresponding result for the resonant $E 2=C 2=0$; long-dashed: fit of RPI $[19,26]$; dot-dot-dashed: corresponding result for the resonant $C 2=0$; short-dashed: "deformed" prediction of SL [18,25]; dotted: "nondeformed" prediction of SL. The shaded band depicts the value of the systematic error. 


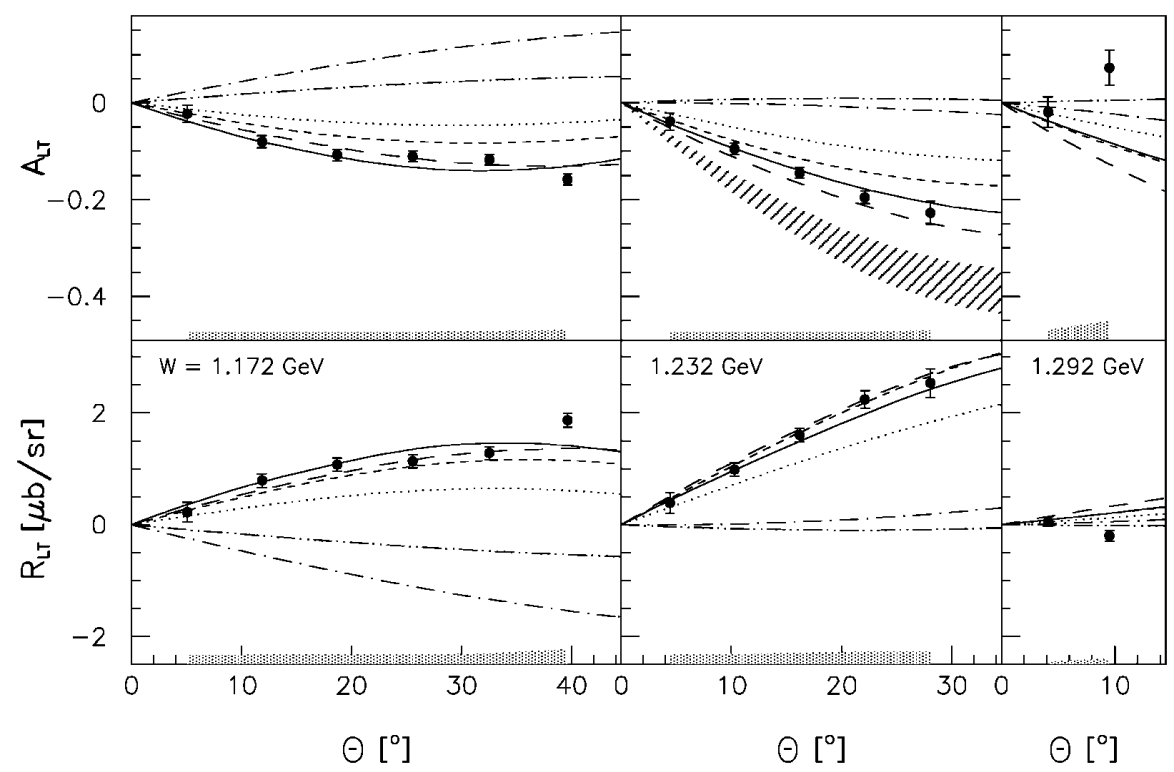

FIG. 2. The longitudinal-transverse asymmetry and response, as a function of the proton polar angle relative to $\vec{q}_{c m}$. The curves and the shaded bands are explained in Fig. 1. The hatched band is the projection of the Bonn result [14].

$\Delta$ resonance and in the method of unitarization. The solid curve is the fit of the MAID-2000 code, where the five parameters controlling the electromagnetic couplings of the $\Delta(1232)$ and of the $\mathrm{P}_{11}(1440)$ (Roper) resonances were fitted to our data [24]. The long-dashed curve results by adjusting all seven free parameters of the RPI model [26], following the same procedure reported in [15], while the short-dashed curve results by judiciously adjusting (without $\chi^{2}$ minimization) the parameters of the SL model to the data [25]. The resulting fits are virtually unchanged if the $P_{n}$ datum [22] is included [24,26].

It has become standard practice in the field, e.g., [9,15], to readjust the parameters of the models by considering only the new data and not the entire data base (enlarged with the new data) through which the models were initially calibrated. It is assumed that the models provide only a local (in $Q^{2}$ ) solution-i.e., no particular form for the $Q^{2}$ evolution of the transition amplitudes is assumed. Thus the pionic data are consistently included via the unitarization procedure $[17,19]$, while the older and less accurate elec- troproduction data are superseded by the far more precise, but consistent, new data.

In Figs. 1 and 2, all calculations properly obtain the position of the cross section maximum; they differ in their detailed shape and in magnitude. The adjusted MAID2000 and RPI models provide an excellent description of the data shown in the figures, with the possible exception of the high $W$ points in Fig. 2.

The $A_{L T}$ and $R_{L T}$ results (Fig. 2) amply demonstrate the sensitivity of our data to the presence of resonant quadrupole amplitudes. All three models fail dramatically if the resonant quadrupole amplitudes are set to zero. However, when the quadrupole strength is adjusted, good agreement is achieved.

The sensitivity of our data to the quadrupole amplitudes allows for the determination of $R_{\mathrm{EM}}$ and $R_{\mathrm{SM}}$ either through a variant of the M1-dominance truncated multipole expansion ("TME") fit (as in [11-14]) or through model extraction, as in [15]. The derived values for the isospin-mixed multipoles are shown in Table I. In TME

TABLE I. Multipoles extracted from the present data at $Q^{2}=0.126(\mathrm{GeV} / c)^{2}$ and $W=$ $1232 \mathrm{MeV}$. Statistical and systematic errors are added quadratically. The measured $P_{n}=$ $-0.40 \pm 0.06$ at $W=1231 \mathrm{MeV}[22]$.

\begin{tabular}{ccccc}
\hline \hline Model & $\begin{array}{c}\left|M_{1+}\right| \\
\left(10^{-3} / m_{\pi^{+}}\right)\end{array}$ & $\begin{array}{c}\operatorname{Re}\left(E_{1+} / M_{1+}\right) \\
(\%)\end{array}$ & $\begin{array}{c}\operatorname{Re}\left(S_{1+} / M_{1+}\right) \\
(\%)\end{array}$ & $P_{n}$ \\
\hline TME (a) & $25.1 \pm 0.7$ & 0 & $-7.6 \pm 0.5$ & $\ldots$ \\
TME (b) & $24.5 \pm 1.6$ & $+0.9 \pm 1.7$ & $-8.5 \pm 1.5$ & $\ldots$ \\
TME (a) & $25.1 \pm 0.7$ & 0 & $-7.6 \pm 0.8$ & $\ldots$ \\
TME (b) & $24.5 \pm 1.1$ & $+0.9 \pm 1.4$ & $-8.5 \pm 1.2$ & $\ldots$ \\
RPI & $25.4 \pm 0.3$ & $+0.8 \pm 0.8$ & $-9.1 \pm 0.8$ & -0.12 \\
MAID & $26.6 \pm 0.2$ & $-2.2 \pm 0.2$ & $-6.7 \pm 0.2$ & -0.51 \\
SL & 27.7 & -3.3 & -4.3 & -0.26 \\
\hline \hline
\end{tabular}


fit (a), as in [14], it is assumed that only the multipoles $M_{1+}$ and $S_{1+}$ contribute and that $R_{L}$ is insignificant. Then we obtain $\operatorname{Re}\left(S_{1+} / M_{1+}\right)=\left(-7.6 \pm 0.3_{\text {stat }} \pm 0.7_{\text {sys }}\right) \%$. The hatched band in Fig. 2 shows the projected asymmetry ( $1 \sigma$ confidence) for our angular range if the Bonn $\operatorname{Re}\left(S_{1+} / M_{1+}\right)=\left(-12.7 \pm 1.5_{\text {stat }}\right) \%$ [14] is adopted. Our data points lie several standard deviations away. Noting that $A_{L T}$ was measured in [14] near $\theta=\pi$, the discrepancy may indicate that terms having a different dependence on $\theta$ than those included in TME fit (a) contribute significantly. If all three $1^{+}$multipoles are adjusted, setting $\left|S_{1+}\right|^{2}=\left|E_{1+}\right|^{2}=\operatorname{Re}\left(S_{1+}^{*} E_{1+}\right)=0$, the derived value of $\operatorname{Re}\left(S_{1+} / M_{1+}\right)$, labeled with "TME (b)" in Table I, is noticeably larger, although not incompatible with, the value extracted through TME fit (a). This is a manifestation of the significant truncation error that characterizes the TME approach.

$\operatorname{Re}\left(S_{1+} / M_{1+}\right)$ and $\operatorname{Re}\left(E_{1+} / M_{1+}\right)$ values are also obtained from the fits of the MAID-2000 [24] and RPI [26] models and from the adjustment of the SL model [25]. While all three models achieve a reasonable agreement with the unpolarized data (Figs. 1 and 2), the resulting values of $P_{n}$ disagree with each other (Table I) and with the experimental value $P_{n}=-0.397 \pm 0.055_{\text {stat }} \pm 0.009_{\text {sys }}$ [22]. The MAID-2000 value could be considered as providing a fair agreement, lying within two standard deviations from the experimental value.

Given the overall success of the MAID-2000 model fit in accounting for our data, we adopt its values of $R_{\mathrm{SM}}=$ $\left(-6.5 \pm 0.2_{\text {stat }+ \text { sys }}\right) \%, R_{\mathrm{EM}}=\left(-2.1 \pm 0.2_{\text {stat }+ \text { sys }}\right) \%$, and $\left|M_{1+}^{3 / 2}\right|=\left(39.8 \pm 0.3_{\text {stat }+ \text { sys }}\right) \times 10^{-3} / m_{\pi^{+}}$for the pure isospin-3/2 multipoles. The statistically incompatible values provided by the other two, equally sophisticated, model analyses indicate that the results are characterized by substantial model uncertainty. The quantification of this uncertainty for each one of the available models is urgently needed. It could remove the apparent contradictions among the available models. We assume that the scatter of the extracted values provides an estimate of the model uncertainty. We therefore attribute, conservatively, to $R_{\mathrm{SM}}$ and $R_{\mathrm{EM}}$ model uncertainties of $\pm 2.5 \%$ and $\pm 2.0 \%$, respectively, and to $\left|M_{1+}^{3 / 2}\right|$ a model uncertainty of $\pm 2.0 \times 10^{-3} / m_{\pi^{+}}$. Previously published $R_{\mathrm{SM}}$ and $R_{\mathrm{EM}}$ results [11-15] have not taken into account this uncertainty. They are subject to comparable model error. This added uncertainty may remove all known inconsistencies among them, when properly estimated.

The data presented here exhibit unprecedented sensitivity to the presence of resonant quadrupole amplitudes. Their analysis leads us to the following conclusions: (i) Extractions of quadrupole strengths based on TME fits are characterized by substantial truncation error and lead to inconsistent results; (ii) claims of large $R_{\mathrm{SM}}$ at low $Q^{2}$ derived from earlier [20] and recent [14] measurements cannot be supported; (iii) even when conservative estimates of systematic and model uncertainties are taken into consideration, an unambiguously negative value for $R_{\mathrm{SM}}$ is obtained. This value supports the claims for an oblate deformed $\Delta$; and (iv) the available pertinent electroproduction models are on the verge of successfully describing the high precision data that are now emerging. It is important that the model errors due to input parameters and model assumptions be quantified. It is essential that measurements be performed that are sensitive to background amplitudes, along with those that are primarily sensitive to quadrupole amplitudes.

We are indebted to Dr. S. S. Kamalov, Dr. D. Drechsel, Dr. L. Tiator, Dr. R. M. Davidson, Dr. N. C. Mukhopadhyay, Dr. T.-S. H. Lee, Dr. T. Sato, and Dr. J. M. Laget for providing us with detailed calculations and valuable comments concerning their models and the issue of "nucleon deformation."

[1] S. L. Glashow, Physica (Amsterdam) 96A, 27 (1979).

[2] S. Capstick and G. Karl, Phys. Rev. D 41, 2767 (1990); S. Capstick and B.D. Keister, Phys. Rev. D 51, 3598 (1995).

[3] G. K. N. Isgur et al., Phys. Rev. D 25, 2394 (1982).

[4] C. E. Carlson, Phys. Rev. D 34, 2704 (1986).

[5] A. J. Buchmann et al., Phys. Rev. C 55, 448 (1997).

[6] D. H. Lu et al., Phys. Lett. C 55, 3108 (1997).

[7] S. S. Kamalov and S. N. Yang, Phys. Rev. Lett. 83, 4494 (1999).

[8] G. C. Gellas et al., Phys. Rev. D 60, 54022 (1999).

[9] G. Blanpied et al., Phys. Rev. Lett. 79, 4337 (1997).

[10] R. Beck et al., Phys. Rev. C 61, 35204 (2000).

[11] K. Batzner et al., Nucl. Phys. B76, 1 (1974).

[12] R. Siddle et al., Nucl. Phys. B35, 93 (1971).

[13] J. C. Alder et al., Nucl. Phys. B46, 573 (1972).

[14] F. Kalleicher et al., Z. Phys. A 359, 201 (1997).

[15] V. V. Frolov et al., Phys. Rev. Lett. 82, 45 (1999).

[16] C. N. Papanicolas, in Topical Workshop on Excited Baryons (World Scientific, Singapore, 1989); A. M. Bernstein et al., Phys. Rev. C 47, 1274 (1993).

[17] D. Drechsel et al., Nucl. Phys. A645, 145 (1999).

[18] T. Sato and T.-S. H. Lee, Phys. Rev. C 54, 2660 (1996).

[19] R. M. Davidson et al., Phys. Rev. D 43, 71 (1991); R. M. Davidson and N. C. Mukhopadhyay, Phys. Lett. B 353, 131 (1995).

[20] R. L. Crawford, Nucl. Phys. B28, 573 (1971).

[21] D. Drechsel and L. Tiator, J. Phys. G 18, 449 (1992).

[22] G. A. Warren et al., Phys. Rev. C 58, 3722 (1998).

[23] C. Mertz, Ph.D. thesis, Arizona State University, 1998 (unpublished); C. Vellidis, Ph.D. thesis, University of Athens (to be published).

[24] S. S. Kamalov (private communication).

[25] T. Sato and T.-S.H. Lee (private communication); (to be published).

[26] R. M. Davidson (private communication). 